\title{
Unilateral cyanosis on tongue as an unusual appearance in emergency department: a case report
}

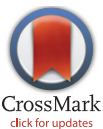

\author{
Seyed Reza Habibzadeh', Esmaeal Rayat Dost ${ }^{2}$, Saeed Barazandehpour ${ }^{3}$, Mahdi Foroughian $^{1 *}$ \\ 'Department of Emergency Medicine, Faculty of Medicine, Mashhad University of Medical Sciences, Mashhad, Iran \\ 2Department of Emergency Medicine, Jahrom University of Medical Sciences, Jahrom, Iran \\ ${ }^{3}$ Department of Emergency Medicine, Kerman University of Medical Sciences, Kerman, Iran
}

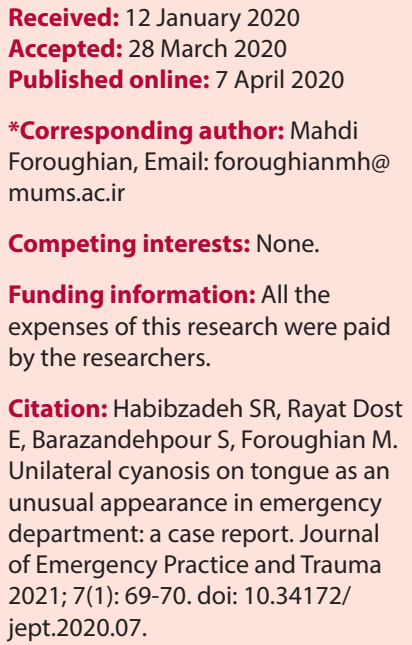

Citation: Habibzadeh SR, Rayat Dost E, Barazandehpour S, Foroughian M. Unilateral cyanosis on tongue as an unusual appearance in emergency department: a case report. Journal of Emergency Practice and Trauma 2021; 7(1): 69-70. doi: 10.34172/ jept.2020.07

\begin{abstract}
Introduction: Unilateral and bilateral tongue cyanosis usually occurs due to the Raynaud syndrome and in the underlying severe types of vasculitis and rheumatology.

Case Presentation: The present study was conducted on a 54-year-old woman who referred to the emergency department with complaints of sudden and painless discoloration of the left half of the tongue. The patient had no history of disease other than diabetes controlled with glibenclamide. Clinical examination of the head and neck revealed evidence of unilateral cyanosis in the left half of the tongue without pain, whose discoloration did not improve with warming of the tongue.

Conclusion: Cyanosis in the emergency department can be managed appropriately by considering some parameters including history taking, history of cyanosis occurrence, history of cardiopulmonary disease, cold sensitivity and history of rheumatologic diseases, presence or absence of nail clubbing, arterial blood oxygen saturation and arterial blood gas test results. These parameters can be effective in designing a treatment regimen, while differentiating the causes of central from peripheral cyanosis.

Keywords: Cyanosis, Tongue, Emergency department
\end{abstract}

\section{Introduction}

Cyanosis refers to the abnormal blue discoloration of the body mucous membranes caused by an increase in the level of deoxygenated hemoglobin. This discoloration is better evident in tissues with thinner epidermis such as lips, oral mucosa, tongue, and ears (1). Unilateral or bilateral cyanosis of the tongue usually occurs due to the Raynaud syndrome and in the underlying severe types of vasculitis and rheumatology (2). Among these, the incidence of unilateral isolated cyanosis on the tongue is one of the rare manifestations in the emergency department which can develop in the context of acute arterial occlusion (2).

\section{Case Presentation}

The patient is a 54-year-old woman who referred to the emergency department with complaints of sudden and painless discoloration of the left half of the tongue. She had a full consciousness and acceptable verbal response, with vital signs on admission as follows: Blood pressure (BP): 140/80 $\mathrm{mm} \mathrm{Hg}$, heart rate (HR): $85 / \mathrm{min}$ (reg), respiratory rate $(\mathrm{RR}): 14 / \mathrm{min}$, temperature $(\mathrm{T}): 37^{\circ} \mathrm{C}$ (axillary), saturation of peripheral oxygen (Spo2): 99\%
(RA) and blood sugar (BS): $210 \mathrm{mg} / \mathrm{dL}$.

The patient had no history of disease other than diabetes controlled with glibenclamide. Clinical examination of the head and neck revealed evidence of unilateral cyanosis in the left half of the tongue without pain, whose discoloration did not improve with warming of the tongue. The tongue movement was not limited and painful (Figure 1). The patient had no uvular edema. Examination of the organs showed no evidence of cyanosis or nail clubbing. Heart and lung auscultation was normal. Examination of other systems was not positive. In addition, examination of the surface of the body and the extremities, except for a few flat warts in the right armpit area, was not positive. The initial tests were ordered for the patient and the results were not abnormal. Color-Doppler ultrasound of the tongue was requested to investigate possible lingual artery occlusion, the findings of which were quite normal. Approximately three hours after admission to the emergency department, the patient's unilateral discoloration was resolved. Due to the rule out of critical causes, airway openness and normality of the initial examinations in the emergency department, the 


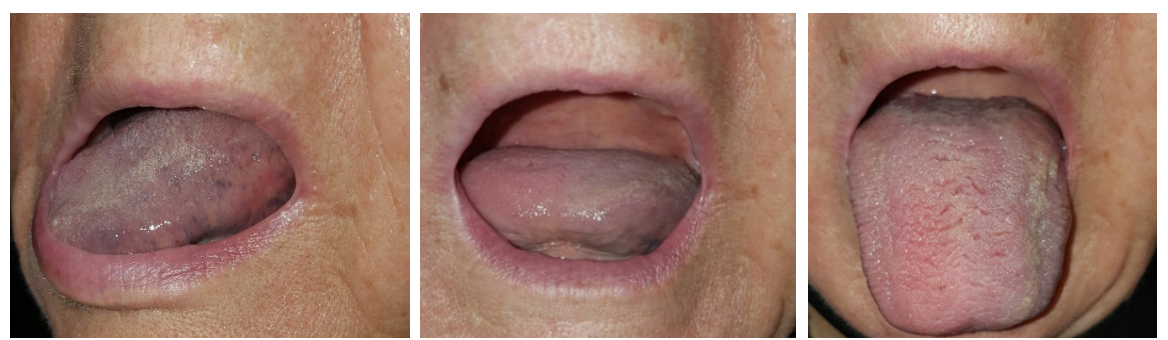

Figure 1. Unilateral cyanosis on tongue in our patient.

patient was discharged from the emergency department on the recommendation of a rheumatologist.

\section{Discussion}

Cyanosis is an important symptom in the emergency department that needs to be carefully examined. In general, cyanosis is more manifested in tissues with thinner epidermis and more visible vessels. In the emergency department, the cyanosis is divided into two main types: central and peripheral. In the central cyanosis, the arterial oxygen saturation is low for a variety of reasons, the most common being cardiopulmonary etiology, causing discoloration of the mucosa of the body, especially the tongue and the oral cavity. In the peripheral cyanosis, the arterial oxygen saturation is normal, but the oxygenation will be lower in the end tissues due to peripheral vasoconstriction, thereby resulting in the cyanosis of the extremities (1). However, the unilateral cyanosis of the tongue without evidence of cyanosis of other mucosa or extremities is a very rare manifestation in the emergency department and only occurs in some underlying severe disorders and vasculitis. On the other hand, the acute occlusion of the lingual artery and vein may occasionally exhibit such presentation (2). Cyanosis in the emergency department can be managed appropriately by considering some parameters including history taking, history of cyanosis occurrence, history of cardiopulmonary disease, cold sensitivity and history of rheumatologic diseases, presence or absence of nail clubbing, arterial blood oxygen saturation and arterial blood gas test results. These parameters can be effective in designing a treatment regimen, while differentiating the causes of central from peripheral cyanosis $(3,4)$.

\section{Conclusion}

Cyanosis of tongue is a rare manifestation of rheumatologic disorders as vasculitis or occlusion of lingual artery and treatment is based on treatment of underlying disease.

\section{Authors' contributions}

All authors contributed equally to study design, drafting article, reading critically and accepted finally proof.

\section{Ethical issues}

Informed consent form was obtained from the patient for the publication of this report.

\section{Acknowledgements}

We would like to thank the Clinical Research Development Unit of peymanieh Educational and Research and Therapeutic Center of jahrom University of Medical Sciences for providing facilities to this work.

\section{References}

1. McMullen SM, Patrick W. Cyanosis. Am J Med 2013; 126(3): 210-2. doi: 10.1016/j.amjmed.2012.11.004.

2. Cohen JC, Palomba ML, Morris LG. Raynaud's phenomenon of the tongue. J Rheumatol 2013; 40(3): 336. doi: 10.3899/ jrheum.121093.

3. Kasper D, Fauci A, Hauser S, Longo D, Jameson JR, Loscalzo J. Harrison's Principles of Internal Medicine. 19th ed. New York: McGraw-Hill Medical Publishing Division; 2016.

4. Marrie TJ, Brown N. Clubbing of the digits. Am J Med 2007; 120(11): 940-1. doi: 10.1016/j.amjmed.2007.06.029. 